\title{
Degradation of Two AChR Populations at Rat Neuromuscular Junctions: Regulation in vivo by Electrical Stimulation
}

\author{
J. S. Andreose, ${ }^{1}$ R. Xu, ${ }^{2}$ T. Lømo, ${ }^{3}$ M. M. Salpeter, ${ }^{2}$ and G. Fumagalli' \\ ${ }^{1}$ CNR Center of Cytopharmacology, Department of Pharmacology, University of Milano, 20129 Milano, Italy, ${ }^{2}$ Section of \\ Neurobiology and Behavior, Division of Biological Sciences, Cornell University, Ithaca, New York, and 3Institute of \\ Neurophysiology, University of Oslo, 0317 Oslo, Norway
}

The effect of electrical stimulation on the stability of junctional ACh receptors (AChR) on soleus muscles of Wistar rats was compared to that of denervation and reinnervation. Denervation causes the degradation rate of the slowly degrading $A C h R s\left(R_{s}\right)$ at the neuromuscular junction to accelerate and be replaced by rapidly degrading $A C h R s\left(R_{r}\right)$, while reinnervation restabilizes the accelerated $R_{s}$. Electrical stimulation initiated at the time of denervation prevented the acceleration of the $\mathbf{R}_{\mathbf{s}}$. It could not, however, reverse the effect of denervation if initiated after the AChRs became destabilized, nor could it slow the degradation rate of the $R_{r}$. We conclude that electrical stimulation of denervated muscle downregulates the expression of the $R_{r}$ and prevents the destabilization of $\mathbf{R}_{\mathbf{s}}$.

[Key words: ACh receptor, degradation, electrical stimulation, neuromuscular junction, denervation, muscle]

Studies on regulation of ACh receptor (AChR) stability in mouse muscle have shown that the neuromuscular junction can contain two types of AChRs with respect to metabolic stability: a slowly degrading population $\left(R_{s}\right)$, present at mature innervated junctions $\left(t_{1 / 2} \sim 10 \mathrm{~d}\right)$; and a rapidly degrading population $\left(\mathrm{R}_{\mathrm{r}}\right)$, predominant at denervated end plates $\left(t_{1 / 2} \sim 1 \mathrm{~d}\right)$ (reviewed in Salpeter and Loring, 1985; Salpeter, 1987b). Following denervation, the preexisting $R_{s}$ loses some of its stability in the postsynaptic membrane, acquires an intermediate half-life of 2-4 d (Levitt and Salpeter, 1981; Stanley and Drachman, 1981; Brett et al ., 1982; Bevan and Steinbach, 1983) and, as it degrades, is replaced by $\mathrm{R}_{r}$, still maintaining a constant site density at the neuromuscular junction (Shyng and Salpeter, 1989). The degradation rates of the two junctional AChR populations are regulated differently by the nerve. Following reinnervation of a denervated muscle, any original $R_{s}$ still remaining in the membrane regain their metabolic stability (Salpeter et al., 1986), while the degradation rate of any $R_{r}$ in this membrane is unaffected (Shyng and Salpeter, 1990). Since the long-term effect of reinnervation

\footnotetext{
Received Oct. 30, 1992; revised Jan. 29, 1993; accepted Feb. 22, 1993.

We heartily thank Sigrid Schaller for technical support, Jim O'Malley for helpful discussions, and Deborah Moslehi for help in preparing the manuscript. This work was supported in part by A.S.I. Grant 91-RS-35 and Theleton-Italy, Project NerveMuscle Interactions (G.F.); the Norwegian Research Council for Science and the Humanities (T.L.); NIH Grants NS09315 and GM10422 (M.M.S.); and a shortterm fellowship from the European Neurobiology Network (to J.S.A.).

Correspondence should be addressed to Miriam M. Salpeter, Department of Neurobiology and Behavior, W113 Mudd Hall, Cornell University, Ithaca, NY 14853.

Copyright $@ 1993$ Society for Neuroscience $0270-6474 / 93 / 133433-06 \$ 05.00 / 0$
}

is complete stabilization of AChRs at the end plate, it appears that innervation, in addition to stabilizing $R_{s}$, inhibits expression of new $R_{r}$.

Since innervation leads to both muscle activity and release of trophic factors, the relative role of these two responses in regulating AChR stabilization is of great interest. Muscle activity has been shown to regulate AChR degradation (Avila et al., 1989; Brenner and Rudin, 1989; Fumagalli et al., 1990; Rotzler et al., 1991). Long-term inactivity of innervated muscle caused by a TTX cuff on the nerve (Fumagalli et al., 1990) or other paralytic agents (Avila et al., 1989) was shown to be equivalent to denervation in inducing acceleration of receptor degradation, whereas long-term $(15 \mathrm{~d})$ electrical stimulation of denervated muscles caused the appearance of stable AChRs (Fumagalli et al., 1990).

To obtain more insights into the mechanisms by which the metabolic stability of junctional AChRs is controlled and the possible role played by muscle activity, we have used rat soleus muscles to study the effects of electrical stimulation in vivo on degradation of $R_{r}$ and $R_{s}$ at the neuromuscular junction. This study did not address the degradation of extrajunctional AChRs appearing after denervation. Our results indicate that neither $R_{r}$ nor the accelerated form of $R_{s}$ can be stabilized by electrical muscle stimulation. However, muscle stimulation initiated at time of denervation prevented $R_{s}$ acceleration. The findings support a model in which muscle activity suppresses the expression of $R_{r}$ and inhibits the mechanism responsible for $R_{s}$ acceleration.

\section{Materials and Methods}

The experiments were done on male Wistar rats from Møllegaards Breeding Centre Ltd. (Skensved, Denmark) and, in one series of denervations, on female Sprague-Dawley rats from Camm Research (Wayne, NJ). The Wistar rats were all about $300 \mathrm{gm}$ except in one series of denervation experiments when they were about $100 \mathrm{gm}$, as were the Sprague-Dawley rats. Surgical operations were performed under Equithesin (Rikshospitalet, Oslo, Norway) anesthesia $(0.4 \mathrm{ml} / 100 \mathrm{gm}$, i.p.). Soleus muscles were chronically denervated by cutting, ligating, and deflecting the sciatic nerve in the thigh. In the reinnervation experiments the axons to the solcus muscle were crushed once just distal to the point where the axons to the soleus and the lateral gastrocnemius muscle branch from the tibial nerve in the fossa poplitea. In the muscle stimulation experiments electrodes were implanted on each side of the denervated soleus muscles and connected to external stimulators as previously described (Westgaard and Lomo, 1988). The stimulation patterns were (1) 60 pulses at $100 \mathrm{~Hz}$ every $60 \mathrm{sec}$, (2) 200 pulses at $20 \mathrm{~Hz}$ every $15 \mathrm{sec}$, or (3) 100 pulses at $100 \mathrm{~Hz}$ every $100 \mathrm{sec}$ (Rotzler et al., 1991). For the AChR degradation studies, soleus muscles were injected with $30 \mu \mathrm{l}$ (100 gm rats) or $50 \mu \mathrm{l}$ (300 gm rats) of $1 \mu \mathrm{M}{ }^{125} \mathrm{I}-\alpha$-bungarotoxin (125I-BTX) ( $>200 \mathrm{Ci} / \mathrm{mmol}$; Amersham) either just before denervation 


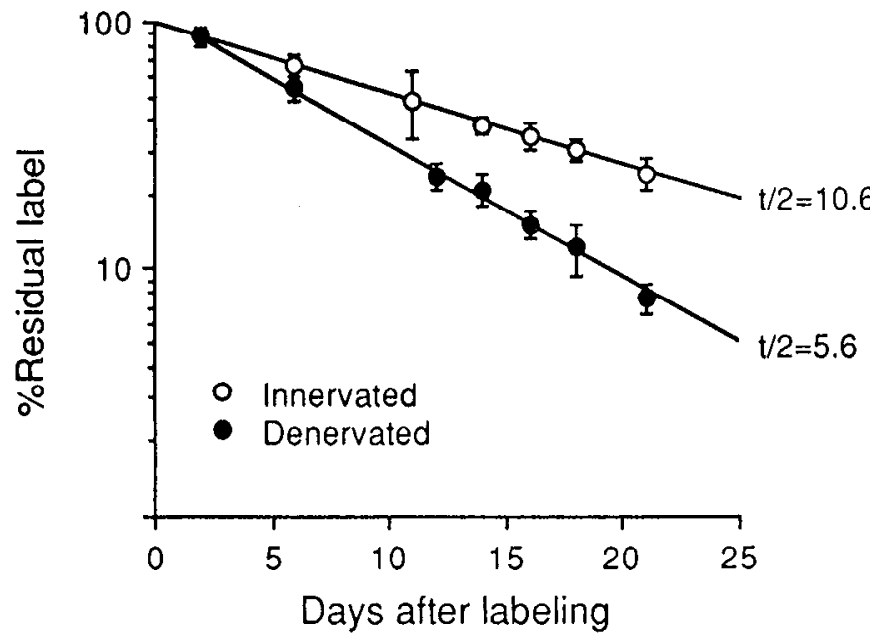

Figure 1. The degradation rate of the $\mathrm{R}_{\mathrm{s}}$ accelerates after denervation. $R_{s}$ receptors were labeled by injection with ${ }^{125}$ I-BTX at the time of dencrvation. End plate-specific radioactivity was measured by gamma counting as described in Materials and Methods and expressed as residual label. The control $(0)$ data were first fitted by linear regression assuming a single exponential. The value extrapolated at day 0 was then set to $100 \%$ and used to normalize the data on the assumption (affirmed experimentally) that all muscles had the same label at the time of the radioactive toxin injection. The data points on day 0 in this and Figures 3 and 4 are thus extrapolated and not experimental values. Half-life values were obtained by linear regression and are shown at the end of the curve. The regression line of the denervated muscles ( $\bullet$ ) starts to diverge from normal (O) 2-5 d after denervation. Values are means \pm SD obtained from three to eight animals at each time point.

for the studies on $R_{s}$, or 10-25 $d$ after denervation for the studies on $R_{r}$ (see Results and the figure captions for more detailed descriptions of the protocols).

At different times after the injections of ${ }^{125}$ I-BTX, three or four animals per time point were anesthetized and perfused through the aorta with $4 \%$ formaldehyde in $0.1 \mathrm{~m}$ phosphate buffer $(\mathrm{pH} 7.4)$. Muscles were then removed and kept in the same fixative solution for $2 \mathrm{hr}$. After staining for acetylcholinesterase, end plate-specific radioactivity was determined either per fiber (Fumagalli et al., 1990) or per muscle (Salpcter ct al., 1986). Briefly, the first method consisted of teasing out thin bundles of muscle fibers (stained for cholinesterase), cutting the bundles into end plate-containing and end plate-free segments, and then gamma counting their radioactivity as well as counting the number of end plates to obtain the specific activity per end plate. The second method consisted of cutting the whole muscle (stained for cholinesterase) into end plate-containing and end plate-free parts, gamma counting the radioactivity of the end plate-containing part, and correcting for the activity of the end plate-free parts on a per weight basis. The degradation rates of the labeled junctional AChR were then calculated by the rate of loss of the end plate-specific radioactivity. Since both methods gave comparable results, the particular method used in each experiment is not further indicated. The gamma counter (Cobra 500, Packard Instruments, Meriden, CT) automatically corrects for the decay rate of the ${ }^{125} \mathrm{I}$. No correction was included for unbinding of the BTX.

The experiments involving chronic muscle stimulation and local application of paralytic toxins have been inspected and permitted by the Norwegian Experimental Board and Ethical Committee for Animal Experiments and were overseen by the veterinarian responsible for the animal house. During the experiments the animals did not appear to suffer pain.

\section{Results}

We first determined how the basic characteristics of AChR degradation in rat soleus muscle compare to those previously established for several mouse muscles.

\section{The degradation rate of rat soleus $R_{s}$ accelerates after denervation}

In one series of experiments ${ }^{125}$ I-BTX was injected into both soleus muscles of $300 \mathrm{gm}$ male Wistar rats and the sciatic nerve on the right side was cut. The soleus muscles were removed 2$18 \mathrm{~d}$ thereafter and end plate-specific radioactivity determined (see Materials and Methods). The degradation rate of the slowly degrading $A C h R s\left(R_{s}\right)$ present at the junction before denervation was $10.6 \pm 1.4 \mathrm{~d}( \pm \mathrm{SD}$; range, 9.4-12.6 d; data from 4 experiments, 3-5 time points per experiment, 3 or 4 animals per time point). About $2 \mathrm{~d}$ after denervation the $\mathrm{R}_{\mathrm{s}}$ accelerated to a halflife of $5.8 \pm 0.7 \mathrm{~d}$ ( $\pm \mathrm{SD}$; range, $5.0-6.8 \mathrm{~d}$; data from 6 separate experiments, 3-5 time points per experiment, 3 or 4 animals per time point) (Fig. 1).

Similar results were obtained in 5-week-old $100 \mathrm{gm}$ rats (data not shown). At denervated and innervated end plates of these young rats the mean half-life of the innervated and accelerated $R_{s}$ was $10.3 \mathrm{~d}$ and $5.0 \mathrm{~d}$, respectively (data from one experiment, four time points, four animals per time point). The acceleration again occurred about $2 \mathrm{~d}$ after denervation, as in the older rats, but the number of binding sites per end plate was less than half the corresponding values in the older rats, indicating that the kinetic behavior of $R_{s}$ was well established even before end plates had reached their full size.

These results show that the $R_{s}$ accelerate earlier but reach a slower degradation rate after denervation in male Wistar rats than in most mouse muscles (Levitt and Salpeter, 1981; Stanley and Drachman, 1981; Bevan and Steinbach, 1983; Wetzel and Salpeter, 1991). To establish whether this difference is related only to a species difference, we performed similar experiments using female Sprague-Dawley rats. The denervation caused the $R_{s}$ to accelerate from a half-life of about $7 \mathrm{~d}$ to about $3.8 \mathrm{~d}$ by 7-9 d after denervation (data not shown). These values are closer to those reported for the mouse. Thus, the time of acceleration and the degradation rate of $R_{s}$ vary between animal strains and, as reported previously, even between different muscles (Bevan and Steinbach, 1983; Wetzel and Salpeter, 1991). The overall response of $R_{s}$ to denervation, however, is very similar in all rodent muscles tested to date. All subsequent experiments were performed only on the $300 \mathrm{gm}$ Wistar rats.

\section{Innervation stabilizes rat soleus $R_{s}$}

For studies on the effect of reinnervation on $R_{s}$ degradation, the axons to the soleus muscle were crushed immediately after they branch off from the tibial nerve in the fossa poplitea. The time course of reinnervalion was determined in a separate series of experiments in which the soleus muscles with the nerve attached were removed from the animal 10-17 $\mathrm{d}$ after the crush and placed in a perfusion chamber. We then examined 30-50 surface fibers with intracellular electrodes for evoked endplate and/or action potentials in response to stimulation of the soleus nerve. No signs of innervation could be detected until day 10 after the crush. On day 12 , end-plate potentials and/or action potentials were evoked in about $40 \%$ of the fibers, and on day 17 , the reinnervation was nearly complete $(>90 \%)$.

The effect of reinnervation on the stability of $R_{s}$ was then determined by injecting ${ }^{125} \mathrm{I}-\mathrm{BTX}$ into the soleus muscles $2 \mathrm{hr}$ before the nerve was crushed and removing muscles at various time points after nerve regeneration, that is, between days 18 and 36 after the crush. Control animals had the nerve cut, ligated, and deflected in the thigh to prevent reinnervation. The 


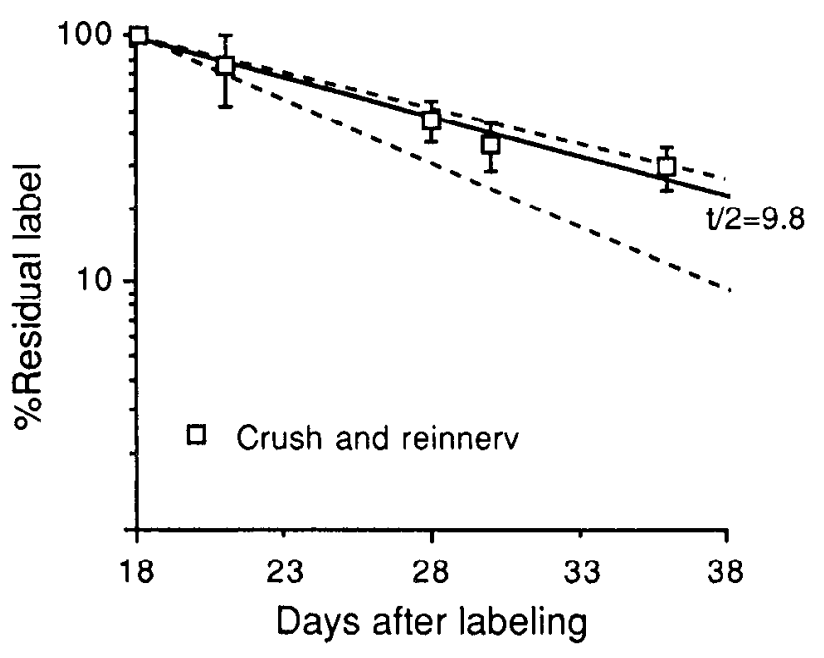

Figure 2. Reinnervation restores stability of the $\mathbf{R}_{\mathrm{s}}$. Muscles were labeled at time of denervation by nerve crush. Residual end plate-specific label (\%) for all groups was normalized to their value obtained experimentally on day 18 after the crush, when more than $90 \%$ of the end plates were reinnervated (as judged by the presence of nerve-evoked end-plate or action potentials in the muscle fibers). Values are mean \pm SD obtained from three to five animals at each time point. Dashed lines give the degradation curves for the $R_{s}$ in innervated and denervated control end plates (see Fig. 1).

data, normalized to the value obtained at day 18 for each experimental group (Fig. 2), demonstrate that, just as in the mouse (Salpeter et al., 1986), reinnervation induces stabilization of the postdenervation accelerated $R_{s}\left(t_{1 / 2}\right.$ of $R_{s}$ at reinnervated muscles $=9.8 \mathrm{~d}$; data from 22 rats).

\section{Electrical stimulation prevents acceleration of $R_{s}$}

Once we had established that the basic characteristics of AChR degradation in rat soleus muscle resembled that of mouse muscle, we examined the effect of electrical muscle activity on this system. $R_{s}$ degradation rate was measured in muscles that were stimulated $(60$ pulses at $100 \mathrm{~Hz}$ every $60 \mathrm{sec}$ ) from the time of denervation until they were removed. No difference was observed between the denervated plus stimulated muscle in one leg and the innervated muscle in the opposite leg (Fig. 3A). These results indicate that $R_{s}$ acceleration does not occur if muscles are kept active from the time of denervation ( 3 time points, 10 rats total).

\section{Electrical stimulation does not stabilize accelerated $R_{s}$}

To determine the effect of muscle activity on the degradation rate of accelerated $R_{s}$, electrical stimulation started $10 \mathrm{~d}$ after denervation and continued until the muscles were removed. The stimulation had no effect on the degradation rate of the accelerated $\mathrm{R}_{\mathrm{s}}$ (Fig. $3 B$ ). The half-life was $5.8 \pm 0.3 \mathrm{~d}( \pm \mathrm{SD}$; average from 2 experiments, 3 time points per experiment, 3 or 4 animals per time point) and was thus the same as the half-life of accelerated $R_{s}$ in denervated, nonstimulated muscles, as given in Figure 1.

\section{Effect of electrical stimulation on $R_{r}$ degradation rate}

Both sciatic nerves were cut, ligated, and deflected in the thigh to avoid regeneration. Twenty-five days later, when most of the $R_{s}$ had degraded and almost all of the receptors at the neuromuscular junction were of the rapidly degrading $\left(R_{r}\right)$ type, radioactive toxin was injected into both muscles. At the same
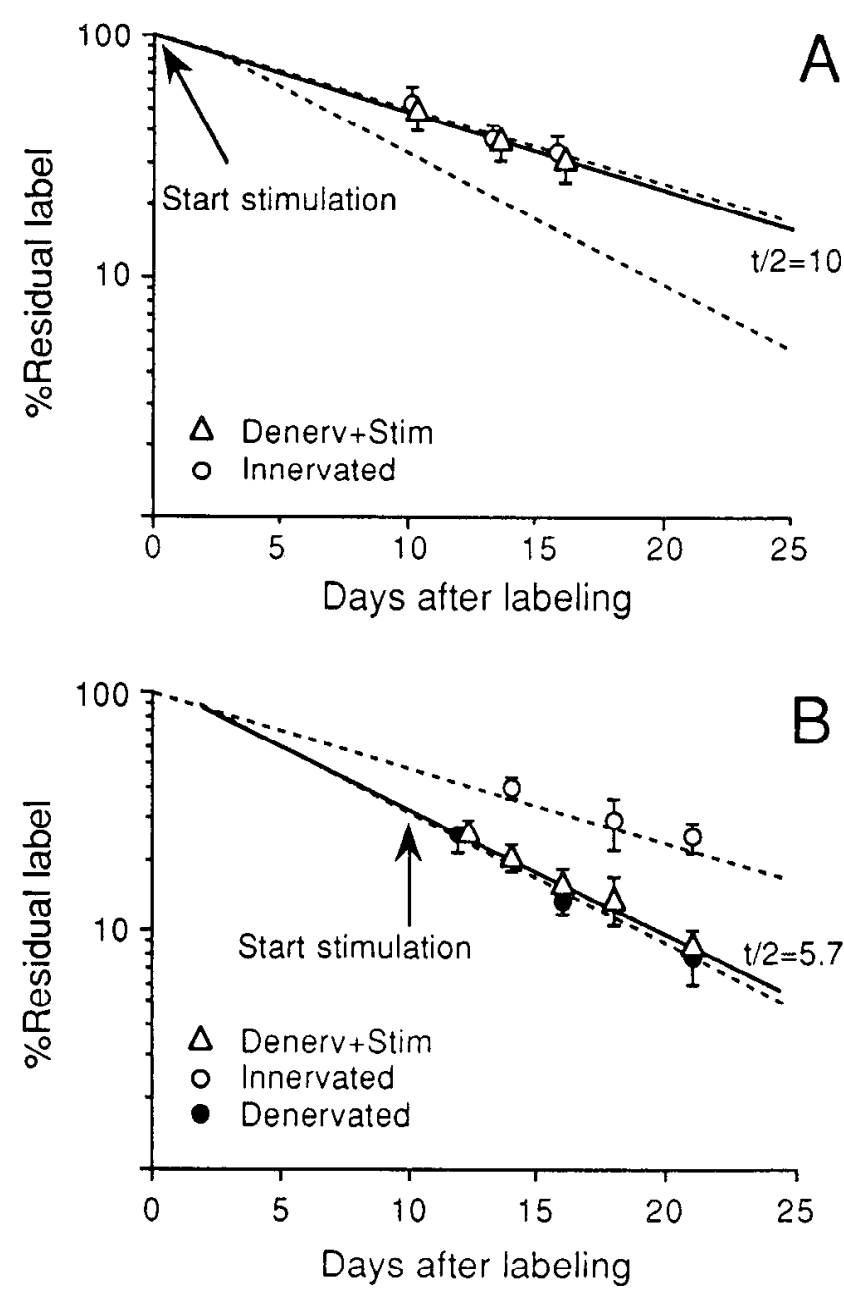

Figure 3. Electrical stimulation prevents acceleration of the $R_{s}$ but does not restabilize the accelerated $R_{s}$. Muscles were injected with ${ }^{175} I-$ BTX before denervation. The muscles in the opposite control leg were either intact $(O)$ or denervated $(\odot)$. The experimental muscles $(\triangle)$ were electrically stimulated with 60 pulses at $100 \mathrm{~Hz}$ every $60 \mathrm{sec}$, the stimulation starting either $<1 \mathrm{hr}$ after denervation $(A)$, or $10 \mathrm{~d}$ later $(B)$. In $A$ the degradation rate is comparable to that in the intact control muscles (upper dashed line), and in $B$, to that in the denervated control muscles (lower dashed line).

time electrodes were implanted and the soleus in the right leg stimulated until both soleus muscles were removed. The stimulation pattern was either 60 pulses at $100 \mathrm{~Hz}$ every $60 \mathrm{sec}$ (Fumagalli et al., 1990) or 200 pulses at $20 \mathrm{~Hz}$ every $15 \mathrm{sec}$. These patterns bear some resemblance to the natural motor unit patterns in fast and slow rat muscles (Hennig and Lomo, 1985; Eken and Gundersen, 1988). Figure $4, A$ and $B$, shows that control $R_{r}$ had a half-life of 2-3 d (somewhat longer than the corresponding values in mouse muscle; Shyng and Salpeter, 1989; Shyng et al., 1991) and that neither stimulation pattern affected the degradation rate of the $R_{r}$.

In the two experiments just described, the stimulation pattern was started long after denervation, when the muscle was considerably atrophied and the number of junctional AChRs was considerably reduced (Frank et al., 1975; Steinbach, 1981; Fumagalli et al., 1990). Therefore, in a third experiment we started the stimulation much earlier, when fibers were less atrophic and the number of junctional AChRs was still normal (Frank et al., 1975; Loring and Salpeter, 1980; Fumagalli et al., 1990). To avoid contaminating our labeled receptors with not yet degraded 

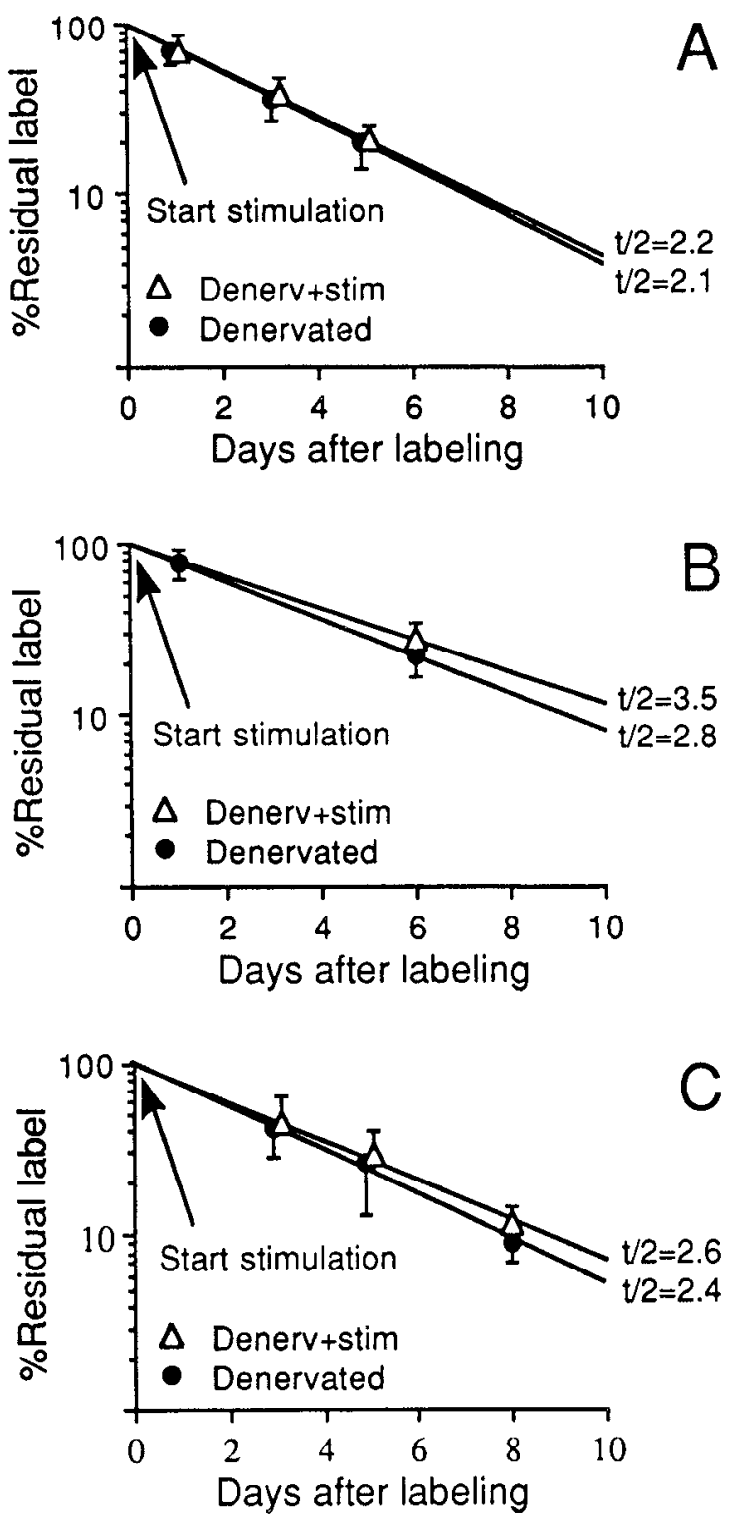

Figure 4. Electrical stimulation does not stabilize the $\mathrm{R}_{\mathrm{r}}$. Soleus muscles in the right and left legs were denervated and injected with ${ }^{125} \mathrm{I}-$ BTX either $25 \mathrm{~d}(A$ and $B)$ or $10 \mathrm{~d}(C)$ later. Electrical stimulation started (as marked by the arrow) $<1 \mathrm{hr}$ after the injection. The values are means \pm SD from at least three muscles per time point and represent percentage of end plate-specific radioactivity remaining, normalized to $100 \%$ on the day of labeling. The half-lives were obtained by linear regression and are shown at the end of the curve for denervated-stimulated $(\triangle)$ and denervated-nonstimulated $(\bullet)$ muscles, respectively. $A$, Twenty-five day denervated muscles were injected with ${ }^{125}$ I-BTX and stimulated with 60 pulses at $100 \mathrm{~Hz}$ every $60 \mathrm{sec}$. $B$. Same as $A$ but the stimulation pattern was 200 pulses at $20 \mathrm{~Hz}$ every $15 \mathrm{sec}$. $C$, Soleus muscles of both legs were denervated and $4 \mathrm{~d}$ later were injected with nonradioactive BTX followed $6 \mathrm{~d}$ later with ${ }^{25} \mathrm{I}$-BTX. The soleus on the right side was then stimulated with 100 pulses at $100 \mathrm{~Hz}$ every 100 sec while the contralateral muscle acted as a nonstimulated control.

$R_{s}$, we inactivated such residual $R_{s}$ by injecting a saturating dose of nonradioactive $\alpha$-BTX $4 \mathrm{~d}$ after denervation. Six days later, ${ }^{125}$ I-BTX was injected to label only newly inserted $R_{r}$, and muscle stimulation was started at that time. In this case, the stimulation pattern was identical to that used by Rotzler et al. (1991) (100 pulses at $100 \mathrm{~Hz}$ every $100 \mathrm{sec}$ ). The soleus muscles were removed 3, 5, and $8 \mathrm{~d}$ later. Again, the stimulation had no effect on the stability of $\mathrm{R}_{\mathrm{r}}$ (Fig. $4 C$ ).

Since the half-life values were not affected by stimulation in any of the different protocols, we conclude that the degradation rate of the $R_{r}$ is insensitive to modulation by muscle activity.

\section{Discussion}

The present study cxamines some of the mechanisms controlling the stability of $\mathrm{AChR}$ degradation rate at Wistar rat soleus neuromuscular junctions. The major results are that (1) denervation causes an acceleration of $R_{r}$ degradation and a replacement of $R_{s}$ by $R_{r}$, as previously described for mouse muscles; (2) reinnervation stabilizes the accelerated $R_{s}$; (3) electrical stimulation starting at the time of denervation keeps $R_{s}$ metabolically stable; and (4) electrical muscle stimulation starting after $R_{s}$ has accelerated does not restabilize $R_{s}$, nor does it stabilize $R_{r}$.

\section{Denervation}

The changes in metabolic properties of the junctional AChR after denervation have previously been described in detail in several mouse muscles (Loring and Salpeter, 1980; Stanley and Drachman, 1981; Levitt and Salpeter, 1981; Brett et al., 1982; Bevan and Steinbach, 1983; Salpeter et al., 1986; Avila et al., 1989; Shyng and Salpeter, 1990). Here we show that denervation of rat soleus muscle similarly accelerates the degradation rate of $R_{s}$ and causes $R_{r}$ to replace $R_{s}$ at the neuromuscular junction. We found, however, that the exact time course of acceleration and the $A C h R$ degradation values in the muscles studied here were somewhat different from those reported previously. In the soleus muscle of male Wistar rat the estimated $t_{1 / 2}$ values for the $R_{r}$ and accelerated form of $R_{s}$ are 2-3 d and 5-6 d, respectively. These values are about twice as long as the corresponding values in mouse muscles and in the female Sprague-Dawley rat soleus muscle. However, even in mouse muscles there is a sizable range of $\mathbf{R}_{\mathrm{s}}$ values (e.g., Bevan and Steinbach, 1983; Shyng and Salpeter, 1990; Wetzel and Salpeter, 1991). Furthermore, the larger $t_{1 / 2}$ for the $R_{r}$ receptors reported here could be due in part to the inclusion of the small $(10 \%)$ slow component synthesized in denervated muscle (Shyng and Salpeter, 1990). In general, many properties of the neuromuscular junction differ in detail among muscles, for example, the number of functional AChRs (Matthews-Bellinger and Salpeter, 1983; Sterz et al., 1983), end-plate size (Fahim et al., 1984; Salpeter, 1987a; Wärhaug, 1992), morphological stability (Lichtman et al., 1987; Wigston, 1989), and interval between time of denervation and onset of $R_{s}$ acceleration. However, the overall response of junctional AChRs to denervation is strikingly similar among different muscles in different species.

\section{Muscle activity and $R_{\mathrm{s}}$}

The fact that reinnervation stabilized the $R_{s}$ motivated us to ask what role muscle activity might play in mediating this effect. Our finding that direct stimulation from the time of denervation prevented acceleration of $R_{s}$ is not surprising since the opposite event (i.e., muscle paralysis) has been shown to accelerate $R_{s}$ (Avila et al., 1989). In addition, it was previously shown that block of nerve conduction by TTX for $18 \mathrm{~d}$ causes the same drop in junctional AChR half-life (from $12 \mathrm{~d}$ to $3 \mathrm{~d}$ ) as does denervation (Fumagalli et al., 1990). The surprising finding in the present work was that direct electrical stimulation, in contrast to reinnervation, failed to restore normal stability to accelerated $R_{s}$. 
It may appear paradoxical that muscle activity can prevent acceleration of junctional $R_{s}$ (present study) and induce expression of new stable $R_{s}$ (as seen in experiments where AChRs are labeled only after periods of electrical stimulation, i.e., Brenner and Rudin, 1989; Fumagalli et al., 1990) but not restabilize old $R_{s}$ once their degradation has accelerated (present study). Our results show that muscle activity cannot fully mimic neural innervation in regulating $R_{s}$ degradation, and suggest that restabilization of $R_{s}$ may require a neurogenic trophic factor independent of muscle activity. At present the nature of such a neurotrophic factor(s) is not known. In nerve-free organ culture, restabilization of $R_{s}$ occurs in the presence of increased levels of cAMP (Shyng et al., 1991). It would be of interest to determine whether cAMP is also involved in the stabilization of $R_{s}$ during normal development in vivo, and whether cAMP and muscle activity interact in this process.

\section{Muscle activity and $R_{r}$}

Our finding that metabolic stability of $R_{r}$ at denervated end plates is unaffected by direct stimulation of the muscle is inconsistent with the finding of Rotzler et al. (1991), who reported that less than $24 \mathrm{hr}$ of muscle stimulation is sufficient to stabilize the junctional AChRs in rat soleus muscles denervated for 1822 d. Although the Rotzler et al. (1991) study did not distinguish between $R_{s}$ and $R_{r}$, the majority of the AChRs after about 20 $d$ of denervation must have been $R_{r}$. The rapid changes in halflife reported by these authors strongly suggest that all the receptors present at time of labeling (the $\mathbf{R}_{\mathrm{r}}$ and the new receptors inserted during the stimulation period) were stabilized by activity. At present, the reasons for the discrepancy with the results of Rotzler et al. (1991) are unclear. We can only point to differences in technique. Rotzler et al. determined the degradation rate of AChRs in vitro over a period that was short relative to the extrapolated half-life, whereas we counted the radioactivity for a considerably longer period in muscles that had been maintained in vivo. Yet in our experiments we could not stabilize the $R_{r}$ with any of three stimulation patterns used, including a pattern identical to that used by Rotzler et al. (1991). Furthermore, electrical stimulation failed to stabilize $R_{r}$ both at a time when the number of $\mathrm{AChRs}$ at the denervated end plate was constant (up to $18 \mathrm{~d}$ after denervation) and at a time when the number of AChRs declined (18-33 d of denervation; see Fumagalli et al., 1990), suggesting that our inability to change the degradation rate of $R_{r}$ was not due to the length of the denervation period. We therefore conclude that the degradation rate of $R_{r}$ at denervated neuromuscular junction is independent of muscle activity. This conclusion was also drawn for $R_{r}$ on muscle cells in culture (see Salpeter et al., 1993).

A previous study had shown that muscle stimulation induces the appearance of slowly degrading $R_{s}\left(t_{1 / 2} \sim 13 \mathrm{~d}\right)$ at long-term denervated end plates (Fumagalli et al., 1990). In that study there was no sign of $R_{r}$ after $15 \mathrm{~d}$ of stimulation. Since our present study showed that neither the $R_{r}$ nor the accelerated $R_{s}$ present in the membrane at the time of labeling could be stabilized by electrical stimulation, we conclude that the stabilization of AChRs at the neuromuscular junction, as seen, for instance, by Fumagalli et al. (1990), involves the degradation of $R_{r}$ molecules during the period of electrical stimulation and their replacement by stable $R_{s}$. Evoked muscle activity thus apparently blocks the expression of new $R_{r}$.

The relative role of neurotrophic factors and muscle activity has been examined for many aspects of neuromuscular orga- nization. In the present study we pursued this question in connection with establishing and maintaining slowly degrading AChRs at vertebrate neuromuscular junction. Our study builds on previous studies that $R_{r}$ could not be stabilized by either innervation (Salpeter et al., 1986) or cAMP (Shyng et al., 1991). It concludes that muscle activity plays a major role in producing a stable AChR population, not by stabilizing AChRs, but by replacing rapidly degrading receptors $\left(R_{r}\right)$ with slowly degrading receptors $\left(R_{s}\right)$ and by maintaining the stability of inserted $R_{s}$. Trophic factors, on the other hand, possibly acting via a mechanism similar to that seen for cAMP analogs, could be involved in stabilizing any accelerated $R_{s}$. (We do not address the additional aspect raised by Ramsay et al., 1992, regarding the stability of $R_{s}$ at the time of insertion.) Several problems, such as the mechanisms whereby trophic factors exert their influence and whether they interact with muscle activity in maintaining a stable AChR population, remain to be solved.

\section{Note added in proof}

After this article was submitted, Coroni et al. (1993) reported stabilization of $R_{r}$ after $6 \mathrm{hr}$ of stimulation at 100 pulses per train at $100 \mathrm{~Hz}$ but not after $4.5 \mathrm{hr}$ at that stimulation pattern, or after $6 \mathrm{hr}$ at 60 pulses per train at $100 \mathrm{~Hz}$, all in vitro. Since we stimulated for much longer periods (days) and obtained no stabilization of $\mathrm{R}_{\mathrm{r}}$ with either of the above patterns in vivo, we have no explanation for why these small differences between a $4.5 \mathrm{hr}$ and $6 \mathrm{hr}$ stimulation or between 60 and 100 pulses per train could produce such dramatic differences when assayed in vitro.

\section{References}

Avila OL, Drachman DB, Pestronk A (1989) Neurotransmission regulates stability of acetylcholine receptors at the neuromuscular junction. J Neurosci 9:2902-2906.

Bevan S, Steinbach JH (1983) Denervation increases the degradation rate of acetylcholine receptors at end-plates in vivo and in vitro. J Physiol (Lond) 336:159-177.

Brenner HR, Rudin W (1989) On the effect of muscle activity on the end-plate membrane in denervated mouse muscle. J Physiol (Lond) 410:501-512.

Brett RS, Younkin SG, Konieczkowski M, Slugg RM (1982) Accelerated degradation of junctional acetylcholine receptor- $\alpha$-bungarotoxin complexes in denervated rat diaphragm. Brain Res 233:133142.

Caroni P, Rotzler S, Britt JC (1993) Calcium influx and protein phosphorylation mediate the metabolic stabilization of synaptic acetylcholine receptors in muscle. J Neurosci 13:1315-1325.

Eken T, Gundersen K (1988) Electrical stimulation resembling normal motor-unit activity: effects on denervated fast and slow rat muscles. J Physiol (Lond) 402:651-669.

Fahim MA, Holley JA, Robbins N (1984) Topographic comparison of neuromuscular junctions in mouse slow and fast twitch muscles. Neuroscience 13:227-235.

Frank E, Gautvik K, Sommerschild H (1975) Cholinergic receptors at denervated mammalian motor endplates. Acta Physiol Scand 99: 66-76.

Fumagalli G, Balbi S, Cangiano A, Lomo T (1990) Regulation of turnover and number of acetylcholine receptors at neuromuscular junctions. Neuron 4:563-569.

Hennig R, Lømo T (1985) Firing patterns of motor units in normal rats. Nature 314:164-166.

Levitt TA, Salpeter MM (1981) Denervated endplates have a dual population of junctional acetylcholine receptors. Nature 291:239241.

Lichtman JW, Magrassi L, Purves D (1987) Visualization of neuromuscular junctions over periods of several months in living mice. $\mathrm{J}$ Neurosci 7:1215-1222.

Loring R, Salpeter MM (1980) Denervation increases turnover rate 
of junctional acetylcholine receptors. Proc Natl Acad Sci USA 77: 2293-2298.

Matthews-Bellinger JA, Salpeter MM (1983) Fine structural distribution of acetylcholine receptors at developing mouse neuromuscular junctions. J Neurosci 3:644-657.

Ramsay DA, Drachman DB, Drachman RJ, Stanley EF (1992) Stabilization of acetylcholine receptors at the neuromuscular synapse: the role of the nerve. Brain Res 581:198-207.

Rotzler S, Schramek H, Brenner HR (1991) Metabolic stabilization of endplate acetylcholine receptors regulated by $\mathrm{Ca}^{2+}$ influx associated with muscle activity. Nature 349:337-339.

Salpeter MM (1987a) Vertebrate neuromuscular junctions: general morphology, molecular organization, and functional consequences. In: The vertebrate neuromuscular junction (Salpeter MM, ed), pp 154. New York: Liss.

Salpeter MM (1987b) Development and neural control of the neuromuscular junction and of the junctional acetylcholine receptor. In: The vertebrate neuromuscular junction (Salpeter MM, ed), pp 55115. New York: Liss.

Salpeter MM, Loring RH (1985) Nicotinic acetylcholine receptors in vertebrate muscle: properties, distribution and neural control. Prog Neurobiol 25:297-325.

Salpeter MM, Cooper DL, Levitt-Gilmour T (1986) Degradation rates of acetylcholine receptors can be modified in the postjunctional plasma membrane of the vertebrate neuromuscular junction. J Cell Biol 103:1399-1403.

Salpeter MM, Andreose J, O'Malley JP, Xu R, Fumagalli G, Lømo T (1993) Degradation of acetylcholine receptors at vertebrate neuromuscular junctions. Ann NY Acad Sci, in press.
Shyng S-L, Salpeter MM (1989) Degradation rate of acetylcholine receptors inserted into denervated vertebrate neuromuscular junctions. J Cell Biol 108:647-651.

Shyng S-L, Salpeter MM (1990) Effect of reinnervation on the degradation rate of junctional acetyicholine receptors synthesized in denervated skeletal muscles. J Neurosci 10:3905-3915.

Shyng S-L, Xu R, Salpeter MM (1991) Cyclic AMP stabilizes the degradation of original junctional acetylcholine receptors in denervated muscle. Neuron 6:469-475.

Stanley EF, Drachman DB (1981) Denervation accelerates the degradation of junctional acetylcholine receptors. Exp Neurol 76:390 396.

Steinbach JH (1981) Neuromuscular junctions and $\alpha$-bungarotoxinbinding sites in denervated and contralateral cat skeletal muscles. J Physiol (Lond) 313:513-528.

Sterz R, Pagala M, Peper K (1983) Postjunctional characteristics of the endplates in mammalian fast and slow muscles. Pfluegers Arch 398:48-54.

Wärhaug O (1992) Species specific morphology of mammalian motor nerve terminals. Anat Embryol (Berl) 185:125-130.

Westgaard RH, Lømo T (1988) Control of contractile properties within adaptive ranges by patterns of impulse activity in the rat. J Neurosci 8:4415-4426.

Wetzel DM, Salpeter MM (1991) Fibrillation and accelerated AChR degradation in long-term muscle organ culture. Muscle Nerve 14: 1003-1012.

Wigston DJ (1989) Remodeling of neuromuscular junctions in adult mouse soleus. J Neurosci 9:639-647. 